www.jmscr.igmpublication.org

Impact Factor 5.84

Index Copernicus Value: 71.58

ISSN (e)-2347-176x ISSN (p) 2455-0450

crossref DOI: _https://dx.doi.org/10.18535/jmscr/v5i9.114

Journal Of Medical Science And Clinical Research

IGM Publication

An Official Publication of IGM Publication

\title{
An Unusual Case of Calcified Hydatid Cyst of Liver Causing Gastric Outlet Obstruction
}

\author{
Authors \\ Prof Dr Sribatsa Kumar Mahapatra ${ }^{1}$, Asst. Prof Dr Pabitra Hembram ${ }^{2}$, \\ Dr Dharbind Kumar $\mathrm{Jha}^{3}$ \\ ${ }^{1}$ Unit Head and Professor, ${ }^{2}$ Assistant Professor, ${ }^{3}$ Post Graduate Student \\ Department of General Surgery, VIMSAR, Burla, Odisha, India \\ Contact number 9437025626, Email: drskm2010@gmail.com
}

\begin{abstract}
Hydatid disease or echinococcosis is a wide spread zoonotic parasite caused by a tape worm Echinococcus granulosus frequently. Liver is commonly involved but calcified hydatid cyst occurs in $10-16 \%$ of cases and calcified hydatid cyst causing gastric outlet obstruction is rare.

We are going to report such a rare case of calcified huge hydatid cyst of liver causing gastric outlet obstruction. A 55yr old women came to opd with complain of vomiting after small amount of meal for 15 days and swelling in right upper and mid abdomen for last 15 years. On examination a mass of size $12 \times 7 \mathrm{~cm}$ was visible in epigastrium and right hypogastrium. The swelling moved with respiration. On palpation liver is enlarged $9 \mathrm{~cm}$ below right costal margin in the right mid clavicular line and a cystic swelling is palpable in relation to the liver $12 x 7-\mathrm{cm}$ in dimension. Nontender. No other mass is palpable. Calcified hydatid cyst was diagnosed in left lobe of liver by ultrasound and CT Scan. Cystopericystectomy done. Post operatively patient recovered well. Huge Calcified hydatid cyst is very rare with gastric outlet obstruction. Surgery is the treatment of choice.

Keywords: Calcified hydatid cyst of liver, gastric outlet obstruction.
\end{abstract}

\section{Introduction}

Hydatid disease, or echinococcosis, is a wide spread zoonotic parasitic disease caused by a tapeworm that continues to be a clinical and public health problem worldwide, especially in areas where animal husbandry and subsistence farming form an integral part of community life. Hydatid disease is most frequently caused by Echinococcus granulosus, and the liver is the most commonly involved organ in two thirds of patients, although it may affect any part of the body, either as a primary or secondary event. The life cycle of Echinococcus requires a definitive host, which is often a dog, and an intermediate host, which is commonly sheep. Humans become accidental intermediate hosts when they become infected after ingesting ova passed in dog feces. The disease occurs principally in sheep-grazing areas, especially where dogs are allowed to stray and eat uncooked viscera. When the parasite reaches the liver parenchyma, it develops into a cystic larval phase, which is visible within 3 weeks and may measure up to $3 \mathrm{~cm}$ in diameter after 3 months. The mature E. granulosus cyst 
consists of three layers: a germinal layer, a laminated layer, and an ectocyst. The inner, germinal layer surrounds the fluid-filled central hydatid cavity and in turn is surrounded by the laminated layer. These two layers together form the endocyst. Compression of the host tissue around the endocyst produces a fibrous layer known as the ectocyst or pericyst. Daughter cysts have a structure similar to the mother cysts, including a laminated and germinative membrane, cyst fluid, brood capsules, and protoscolices. The only difference is the absence of an adventitial layer. The ectocyst or pericyst is a fibrous capsule that develops from host tissue as an inflammatory reaction to E. granulosus. This thick fibrous layer is present in hydatid cysts in the liver and spleen but is absent in pulmonary and brain hydatid cysts. With time, the adventitial layer may calcify, either partially or totally. Calcification of the parasite occurs in about $10 \%$ to $16,6 \%$ of the cases of hepatic echinococcosis, and it may require about 5 to 10 years to develop, being very common in the aged population ${ }^{[6]}$. Hydatid cysts in the liver may cause symptoms as a result of direct pressure, from the inflammatory reaction around the cyst, from distortion of neighboring structures or viscera, or as a result of erosion into the bile duct, pleural space, or peritoneal cavity. Rarely the cyst may communicate with the bronchial tree, pericardium, or digestive tract. We are going to report such rare case of calcified huge hydatid cyst with gastric outlet obstruction.

\section{Case Report}

A $55 \mathrm{yr}$ old women came to opd with complain of vomiting after small amount of meal for 15 days and swelling in right upper and mid abdomen for last 15 years. The swelling gradually increased in size to attend present size. There is no history of pain over the swelling. There is no history of fever.

On examination a mass of size $12 \times 7 \mathrm{~cm}$ was visible in epigastrium and right hypogastrium. The swelling moved with respiration and more prominent in knee elbow position. On palpation liver is enlarged $9 \mathrm{~cm}$ below right costal margin in the right mid clavicular line and a cystic swelling is palpable in relation to the liver $12 \times 7-\mathrm{cm}$ in dimension. Nontender. No other mass is palpable. No free fluid in the abdomen and normal bowel sounds are audible. Other systemic examination is normal. Liver function test normal. Ultrasonography revealed a well defined multiloculated heterogeneously echogenic space occupying lesion of size $15.1 \times 4.9 \times 8.1$ ) vol 530cc is noted in left lobe of liver involving segment ii, iii, iv. Very thinned out hepatic parenchyma. Multiple daughter cysts and echogenic hydatid sand are noted in the liver. Computed Tomography scan revealed a well defined heterogenous 15.3 x 8.5 $\mathrm{cm}$ cystic SOL of peripheral hypodensity and central hyperdensity with internal daughter cyst arising from left lobe of liver extending inferiorly upto L3-4 vertebrae [FIG 1.]. Thick wall and dense wall calcification noted. On oral contrast no enhancement taken. Thus our diagnosis was calcified hydatid cyst of left lobe of liver. Then we planned for conservative surgical excision of cyst. Thus under all available aseptic condition in general anaesthesia a midline incision was given then abdomen cavity packed with cetrimide (savlon) soaked tetra to isolate the cyst and also to prevent spillage. On aspiration of cyst we got dry tap. Then omentum adherent to this cyst was separated out. The cyst compressing the pylorus and $1^{\text {st }}$ part of duodenum was dissected and mobilised. Then carefully a surgical plane just outside the pericyst layer was made without opening the cyst, the parasite and the adventitial layer were excised en bloc. [FIG: 2\&3] Hemostasis maintained. Appendicectomy done. Omental pack given and drain given in right sub hepatic space. Layer wise closure of abdominal wall done. Postoperatively patient recovered well. 


\section{JMSCR Vol||05||Issue||09||Page 28054-28057||September}

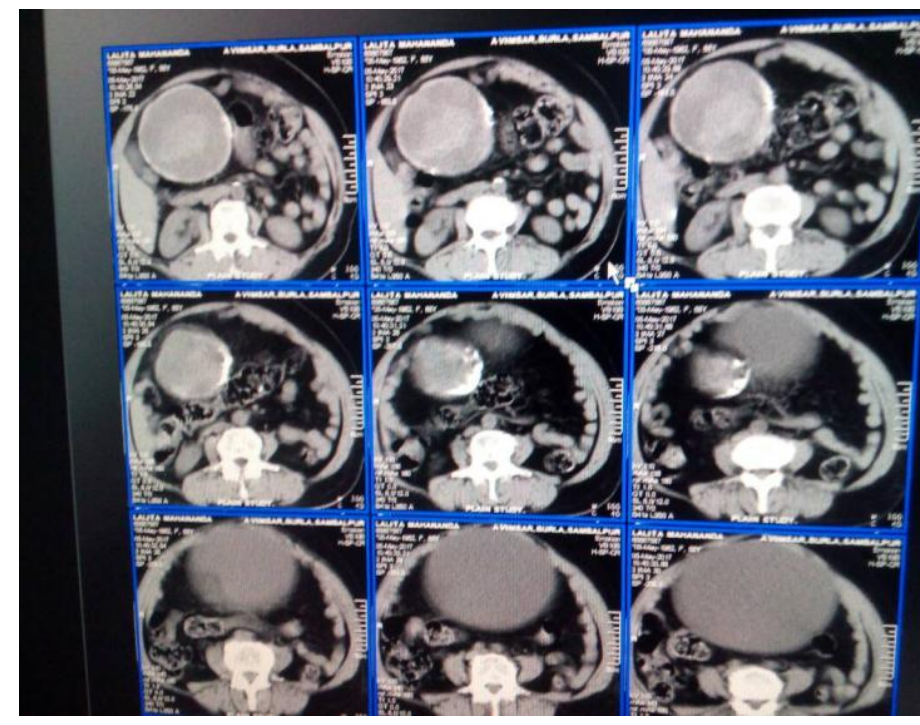

Figure 1: Showing CT scan picture of Hydatid cyst in left lobe of liver

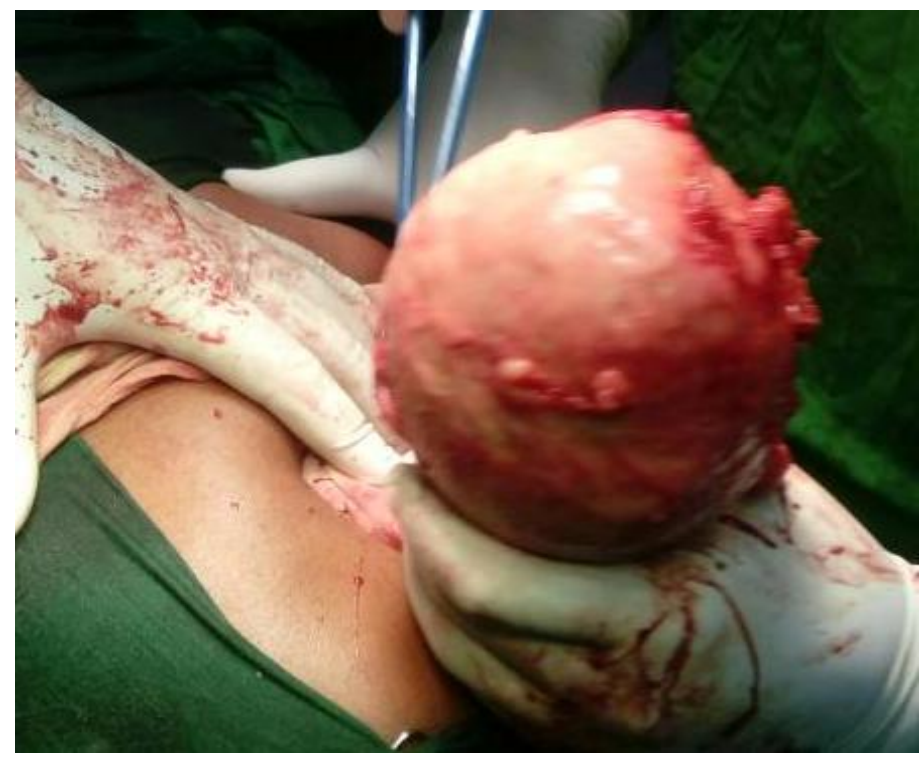

Fig 2: Showing the huge calcified hydatid cyst after cystopericystectomy

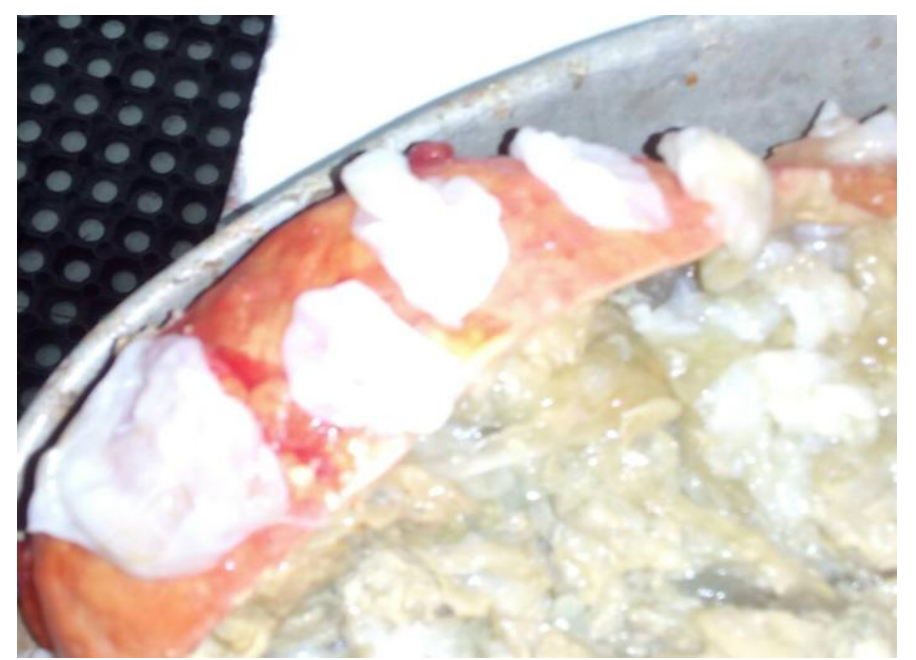

Fig 3: showing the daughter cyst .

\section{Discussion}

Hydatid cysts in the liver may cause symptoms as a result of direct pressure, from the inflammatory reaction around the cyst, from distortion of neighboring structures or viscera, or as a result of erosion into the bile duct, pleural space, or peritoneal cavity. Rarely the cyst may communicate with the bronchial tree, pericardium, or digestive tract. ${ }^{[1]}$ Calcification takes place in 10 $16 \%$ of cases. ${ }^{[6]}$ Usually it takes more than 5 years for calcification in our case the patient has c/o swelling for last 15 years. The cyst is usually biologically inert i.e it does not get infected or rupture to bile duct and abdominal cavity, thorax. In our case we got a dry tap on aspiration which supports the above literature. But in some cases it may get ruptured to bile duct abdominal cavity and thorax but in our case it was inert. The parasite is viable even a part of cyst wall get calcified and the cyst size may increase. In our case there is history of slowly increase in size of cyst for last 15 years. The calcific salts accumulate mainly in the adventitia or sometimes in the wall of mother or daughter cysts with consequent partial or total calcification. Plain radiography of the abdomen is of limited value in the diagnosis of hydatid liver cysts. US and CT are standard investigations used for diagnosis. On ultrasonography we got a well defined multiloculated heterogeneously echogenic space occupying lesion of size $15.1 \times 4.9 \times 8.1$ ) vol $530 \mathrm{cc}$ is noted in left lobe of liver involving segment ii, iii, iv. Thick wall with calcification noted. Multiple daughter cysts and echogenic hydatid sand were noted in the liver. As per The World Health Organization (WHO) Informal Working Group on Echinococcosis (2003) described ultrasound classification system. It was CE5. Computed Tomography scan revealed a well defined heterogenous $15.3 \times 8.5 \mathrm{~cm}$ cystic SOL of peripheral hypodensity and central hyperdensity with internal daughter cyst arising from left lobe of liver extending inferiorly upto L3-4 vertebrae [FIG 1.]. Thick wall and dense wall calcification noted. ${ }^{[1,6,3,4]}$. Medical therapy is of less benefit in 
these cases due to calcification of pericyst the drug cannot enter the cyst and kill metacestode. Thus surgery or wait and watch principle remains the options for treatment. In our case we operated the cyst due to a large cyst with features of gastric outlet obstruction. We used the midline upper abdominal incision for maximum exposure, cyst in left lobe of liver and as well as to explore the adjacent structures as there was features of gastric outlet obstruction. Other incisions used are right subcoastal, thoraco-abdominal and combined upper midline and right subcoastal incision and bilateral subcoastal incision. After that we put cetrimide soaked tetra in the peritoneal cavity. On aspiration from cyst a dry tap was observed. Then we performed cystopericystectomy (i.e by creating a surgical plane just outside the pericyst layer without opening the cyst, the parasite and the adventitial layer are excised en bloc) ${ }^{[1,2,3,4,5]}$. Then hemostasis was maintained and peritoneal toileting done. The stomach and intestines were found to be normal. Omentum was packed in the cavity to obliterate the space as the walls are non collapsible and there is chance of prolonged billiary leaked and infection. A drain was given in right sub hepatic space. Capitonage is usually not possible in such a large cyst. The method of omentum packing and giving drain and giving drain alone is debatable. But Omentum has a natural absorptive capacity that decreases the risk of infection and minimizes fistula formation and it is safer to use it. Due to chances of billiary leak and infection appendicectomy was done to prevent further attack of appendicitis and polymicrobial infection and formation of liver abscess which could worsen the condition. Postoperative course was uneventfull and patient was discharged with full recovery. Perioperative prophylaxis should start 1 week before the procedure. Recommendations for post treatment prophylaxis are 3 to 8 weeks for uncomplicated cases. In complicated cases with a higher risk of spillage of cyst contents, 3 to 6 months of treatment is arbitrarily advised. Thus we gave tablet albendazole $400 \mathrm{mg}$ twice daily for 8 weeks post operatively.

\section{Conclusion}

Huge Calcified hydatid cyst of liver causing gastric outlet obstruction is a very rare disesase. USG and CT scan is must for diagnosis. Surgery is the treatment of choice.

\section{References}

1. Milicevic, M.(1994).Hydatid disease in Blumgart L. H.,Surgery of the LiverandBiliary tract. Vol.II, Churchull Livingstone,pp.1121-1150.

2. Abu Zeid M, et al, 1998: Surgical treatment of hepatic hydatid cysts. Hepatogastroenterology 45:1802-1806

3. Gharbi HA, et al, 1981: Ultrasound examination of the hydatic liver. Radiology 139:459-463

4. Terblanche J, Krige JEJ, 1998: The management of hepatic Echinococcus. In Cameron JL (ed): Current Surgical Therapy, 6th ed. Baltimore, Mosby, pp 326-330.

5. Magistrelli P, et al, 1991: Surgical treatment of hydatid disease of the liver: a 20year experience. Arch Surg 126:518-522.

6. HPB Surg. 1999;11(4):253-9. Surgical management of calcified hydatid cysts of the liver. Prousalidis J(1), Tzardinoglou E, Kosmidis C, Katsohis K, Aletras O. 